\title{
Pneumatosis Cystoides Intestinalis in the Setting of Clindamycin Use: An Association or a Coincidence?
}

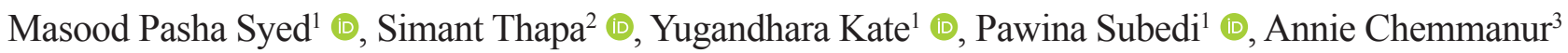 \\ ${ }^{1}$ Clinic of Internal Medicine, Saint Vincent Hospital, Worcester, Massachusetts \\ ${ }^{2}$ Clinic of Pulmonary Critical Care and Sleep Medicine, SUNY Upstate Medical University, Syracuse, NY \\ ${ }^{3}$ Clinic of Gastroenterology, Saint Vincent Hospital, Worcester, Massachusetts
}

\begin{abstract}
A 60-year-old male with a medical history of hemorrhoids presented with persistent watery diarrhea of 4-week duration associated with left lower quadrant abdominal cramps, bloating, and 2 episodes of hematochezia. He was prescribed with clindamycin 6 weeks ago for dental infection, and his symptoms started after taking clindamycin for 1 week. He denied fever, chills, nausea, vomiting, weight loss, or change in appetite. Vital signs were stable and physical examination was significant for mild tenderness in the left lower quadrant. Computed tomography scan of the abdomen showed air-filled cysts involving the proximal to mid-sigmoid colon consistent with pneumatosis cystoides intestinalis (PI) without pneumoperitoneum (Figure 1). Stool Clostridium difficile toxin, stool microscopic examination results, and culture were negative. Colonoscopy showed multiple air-filled cysts of various sizes without any active bleeding (Figure 2). He was managed conservatively without antibiotic agents and his diet was advanced gradually as tolerated. A repeat colonoscopy after 3 months showed mild improvement but abdominal pain and diarrhea had resolved. He was advised for a follow-up colonoscopy in 1 year.
\end{abstract}

PI signifies the presence of gas within the wall of the small or large intestine. Its presentation can range from an incidental finding in an asymptomatic patient that can resolve spontaneously to a severe case of intestinal necrosis that may require surgery (1).

The etiology of PI seems to be multifactorial, although the exact cause is unknown. Many theories have been proposed as follows:

- Mechanical theory: Increased pressure owing to bowel obstruction or emphysema can cause gas to dissect into the bowel wall from either the intestinal lumen or the lungs via the mediastinum.

- Bacterial theory: The gas found within the bowel wall may be caused by gas-forming bacteria that then enter the submucosa through mucosal rents or increased mucosal permeability.

- Biochemical theory: Gas produced by fermentation of car-

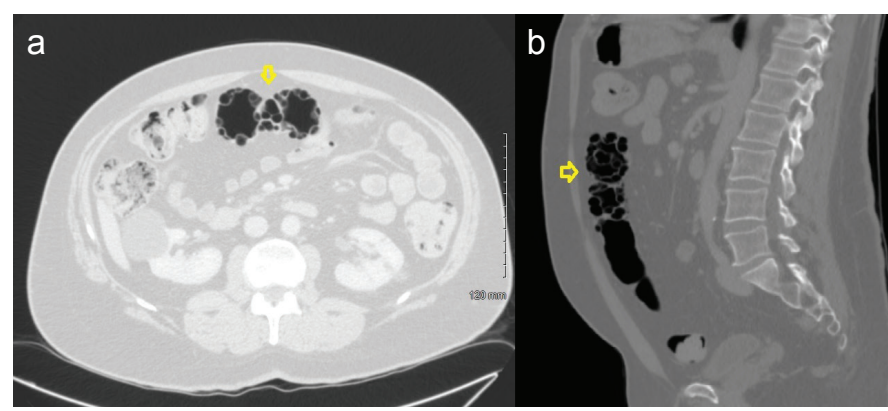

FIG. 1. a, b. Cross-sectional view (a) and sagittal view (b). Computed tomography scan of abdomen showing air-filled cysts (arrow) involving the proximal to mid-sigmoid colon consistent with pneumatosis cystoides intestinalis.

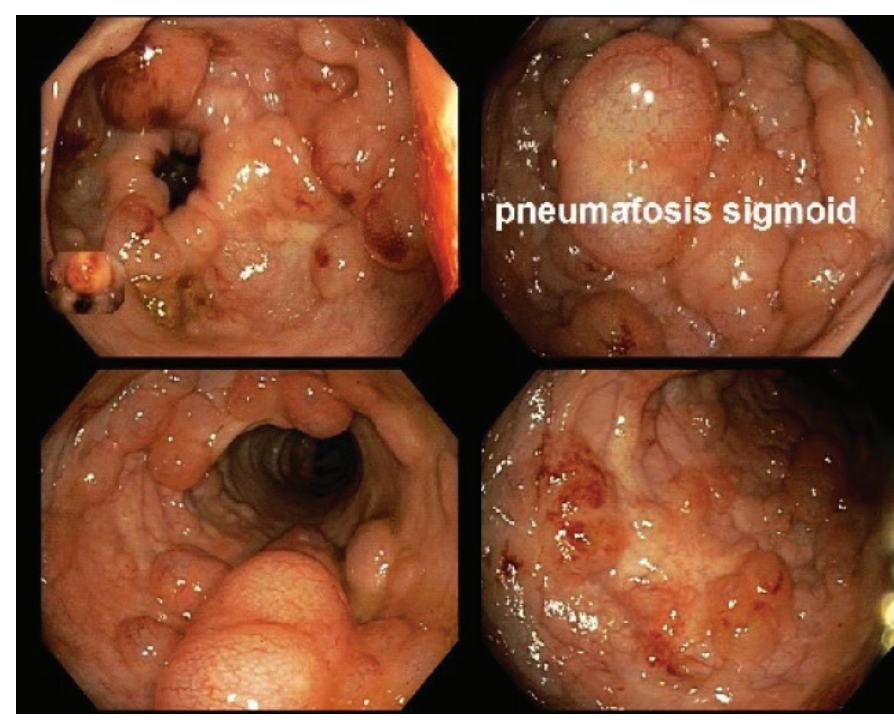

FIG. 2 Colonoscopy showing multiple air-filled cysts of different sizes in the sigmoid colon.

Address for Correspondence: Masood Pasha Syed, Clinic of Internal Medicine, Saint Vincent Hospital, Worcester, Massachusetts

e-mail: dr.syedmasoodpasha@gmail.com

Received: March 12, 2020 Accepted: July 3, 2020 •DOI: 10.4274/balkanmedj.galenos.2020.2020.3.69

Available at www.balkanmedicaljournal.org

ORCID iDs of the authors: M.P.S. 0000-0002-3252-9094; S.T. 0000-0001-6651-1397; Y.K. 0000-0002-7790-4396; P.S. 0000-0003-1969-5618.

Cite this article as:

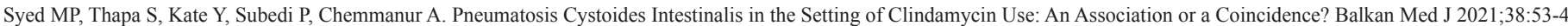

Copyright@Author(s) - Available online at http://balkanmedicaljournal.org/ 
bohydrates by the luminal bacteria may be forced directly through the mucosa trapping it in the submucosa.

- Drug-induced theory: Caused by the loss of mucosal integrity allowing intraluminal gas to escape into the bowel wall (2).

Complications related to PI include bowel obstruction, volvulus, intussusception, hematochezia, and pneumoperitoneum if the subserosal cysts rupture. Mild cases of PI can be managed conservatively with or without antibiotic agents but severe cases with life-threatening complications (intestinal perforation, pneumoperitoneum, intestinal necrosis) need immediate surgical intervention (3).

In our case, the etiology could be a combination of the various theories mentioned earlier. To the best of our knowledge, our case is the first reported possible association of PI with clindamycin. We understand that association does not necessarily mean causation; however, the timeline fits his symptoms, and owing to a lack of any other explanation, we concurred that this may be the cause. Further such cases need to be reported to increase the strength of this association and to better understand the exact pathogenesis.

Informed Consent: Due to the ongoing pandemic, authors did not call the patient to the hospital to take physical consent and took verbal consent instead on the phone.

Author Contributions: Concept - M.P.S., S.T., Y.K., P.S., A.C.; Supervision - M.P.S., S.T., Y.K., P.S.; Data Collection and/or Processing - M.P.S., S.T., Y.K., P.S.; Analysis and/or Interpretation - M.P.S., S.T., Y.K., P.S., A.C.; Literature Search - M.P.S., S.T., A.C.; Writing Manuscript - M.P.S., S.T., Y.K., P.S.; Critical Review - M.P.S., S.T., Y.K., P.S.

Conflict of Interest: The authors have no conflicts of interest to declare.

Funding: The authors declared that this study has received no financial support.

\section{REFERENCES}

1. Wang YJ, Wang YM, Zheng YM, Jiang HQ, Zhang J. Pneumatosis cystoides intestinalis: Six case reports and a review of the literature. BMC Gastroenterol 2018;18:100. doi: 10.1186/s12876-018-0794-y. [Crossref]

2. Ho LM, Paulson EK, Thompson WM. Pneumatosis intestinalis in the adult: Benign to life-threatening causes. AJR Am J Roentgenol 2007;188:1604-13. [Crossref]

3. Zorcolo L, Capra F, D'Alia G, Scintu F, Casula G. Pneumatosis cystoides of the right colon: a possible source of misdiagnosis. Report of a case. Chir Ital 2005;57:121-6. 